\title{
A research on the new polynomials involved with the central factorial numbers, Stirling numbers and others polynomials
}

Jung Yoog Kang* and Cheon Seoung Ryoo

"Correspondence:

rkdwjddnr2002@yahoo.co.kr Department of Mathematics, Hannam University, Daejeon, 306-791, Korea

\begin{abstract}
Recently, many mathematicians have studied different kinds of the Euler, Bernoulli, and Genocchi polynomials. In this paper, we give another definition of the polynomials $\widetilde{U}_{n}^{(\alpha)}(x)$. We find some theorems and identities related to polynomials $\widetilde{U}_{n}^{(\alpha)}(x)$ containing the central factorial numbers and Stirling numbers. We also derive interesting relations between the polynomials $\widetilde{U}_{n}^{(\alpha)}(x)$ and the Euler polynomials and the Genocchi polynomials.

Keywords: polynomials $\widetilde{U}_{n}^{(\alpha)}(x)$; Stirling numbers; central factorial numbers; Euler polynomials; Genocchi polynomials
\end{abstract}

\section{Introduction}

The Stirling numbers of the first kind $s(n, k)$ are defined by [1]

$$
\sum_{k=0}^{n} s(n, k) x^{k}=x(x-1)(x-2) \cdots(x-n+1) .
$$

The generating function of (1.1) is as follows:

$$
(\log (1+x))^{k}=k ! \sum_{n=k}^{\infty} s(n, k) \frac{x^{n}}{n !}
$$

From (1.1) and (1.2), we become aware of some properties of the Stirling numbers of the first kind, $s(n, k)[1]$ :

$$
s(n, k)=s(n-1, k-1)-(n-1) s(n-1, k),
$$

with

$$
\begin{aligned}
& s(n, 0)=0 \quad(n \in \mathbb{N}), \\
& s(n, n)=1 \quad\left(n \in \mathbb{Z}^{+}=\mathbb{N} \cup 0\right), \\
& s(n, 1)=(-1)^{n-1}(n-1) ! \quad(n \in \mathbb{N}), \\
& s(n, k)=0 \quad(k>n \text { or } k<0) .
\end{aligned}
$$


We usually define the central factorial numbers $T(n, k)$ by the following expansion formula $[2,3]$ :

$$
\sum_{k=0}^{n} T(n, k) x\left(x-1^{2}\right)\left(x-2^{2}\right) \cdots\left(x-(k-1)^{2}\right)=x^{n} .
$$

The generating function of (1.3) is as follows:

$$
\left(e^{x}+e^{-x}-2\right)^{k}=(2 k) ! \sum_{n=k}^{\infty} T(n, k) \frac{x^{2 n}}{(2 n) !}
$$

By using (1.3) and (1.4), we become aware of some properties of the central factorial numbers $T(n, k)$ :

$$
\begin{aligned}
& T(0,0)=1, \\
& T(n, 0)=0 \quad(n \in \mathbb{N}), \\
& T(n, 1)=1 \quad(n \in \mathbb{N}), \\
& T(n, k)=T(n-1, k-1)+k^{2} T(n-1, k), \quad \text { with } \\
& T(n, 2)=\frac{1}{4}\left(4^{n-1}-1\right), \quad T(n, 3)=\frac{9^{n}}{360}-\frac{4^{n}}{60}+\frac{1}{24} \quad(n \in \mathbb{N}) .
\end{aligned}
$$

The Euler numbers $E_{n}$ and Euler polynomials $E_{n}(x)$ are defined by

$$
\begin{aligned}
& E_{0}=1, \quad E_{n}=-\sum_{k=1}^{\left[\frac{n}{2}\right]}\left(\begin{array}{c}
n \\
2 k
\end{array}\right) E_{n-2 k} \quad(n \geq 1), \\
& E_{n}(x)=\frac{1}{2^{n}} \sum_{k=0}^{\left[\frac{n}{2}\right]}\left(\begin{array}{c}
n \\
2 k
\end{array}\right)(2 x-1)^{n-2 k} E_{2 k} \quad(n \geq 0),
\end{aligned}
$$

where $[x]$ is the greatest integer not exceeding $x[2,4,5]$.

For a real or complex parameter $\alpha$, the generalized Euler polynomials of degree $n$ are defined by the following generating functions:

$$
\sum_{n=0}^{\infty} E_{n}^{(\alpha)}(x) \frac{t^{n}}{n !}=\left(\frac{2}{e^{t}+1}\right)^{\alpha} e^{t x} \quad\left(|t|<\pi ; 1^{\alpha}:=1\right)
$$

Clearly, we have $E_{n}^{(1)}(x)=E_{n}(x)[6-12]$.

The Genocchi polynomials are defined by

$$
\sum_{n=0}^{\infty} G_{n}^{(\alpha)}(x) \frac{t^{n}}{n !}=\left(\frac{2 t}{e^{t}+1}\right)^{\alpha} e^{t x}
$$

with the usual convention of writing $G^{n}(x)$ by $G_{n}(x)$. In the special case, $x=0, G_{n}(0)=G_{n}$ are called the $n$th Genocchi numbers [11, 13-16].

In Section 2, we define polynomials $\widetilde{U}_{n}^{(\alpha)}(x)$. We consider the addition theorem for these polynomials. We also investigate some identities which are related to polynomials $\widetilde{U}_{n}^{(\alpha)}(x)$. 
We also try to find relations between the polynomials $\widetilde{U}_{n}^{(\alpha)}(x)$, the Stirling numbers $s(n, k)$, and the central factorial numbers $T(n, k)$. In Section 3, we derive some special relations of the polynomials $\widetilde{U}_{n}^{(\alpha)}(x)$ and the Euler polynomials. We also find a link between the polynomials $\widetilde{U}_{n}^{(\alpha)}(x)$ and the Genocchi polynomials.

\section{Some properties involving a certain family of polynomials}

In this section, we define the polynomials $\widetilde{U}_{n}^{(\alpha)}(x)$ and study several theorems of the polynomials $\widetilde{U}_{n}^{(\alpha)}(x)$. We can see some interesting properties of the polynomials $\widetilde{U}_{n}^{(\alpha)}(x)$.

Definition 2.1 For $\alpha, x \in \mathbb{C}$, the polynomials $\widetilde{U}_{n}^{(\alpha)}(x)$ are defined by

$$
\sum_{n=0}^{\infty} \widetilde{U}_{n}^{(\alpha)}(x) \frac{t^{n}}{n !}=\left(\frac{e^{t}}{e^{t}+e^{-t}-1}\right)^{\alpha} e^{t x}
$$

Note that $\widetilde{U}_{n}^{(\alpha)}(0)=\widetilde{U}_{n}^{(\alpha)}$. That is, $\widetilde{U}_{n}^{(\alpha)}$ are called the $n$th numbers. By using Definition 2.1, we have the addition theorem of the polynomials $\widetilde{U}_{n}^{(\alpha)}(x)$.

Theorem 2.2 Let $\alpha, x, y \in \mathbb{C}$ and let $n$ be non-negative integers. Then we get

$$
\widetilde{U}_{n}^{(\alpha)}(x+y)=\sum_{l=0}^{n}\left(\begin{array}{l}
n \\
l
\end{array}\right) \widetilde{U}_{n-l}^{(\alpha)}(x) y^{l}
$$

Proof From Definition 2.1, we get

$$
\begin{aligned}
\sum_{n=0}^{\infty} \widetilde{U}_{n}^{(\alpha)}(x+y) \frac{t^{n}}{n !} & =\left(\frac{e^{t}}{e^{t}+e^{-t}-1}\right)^{\alpha} e^{(x+y) t} \\
& =\left(\sum_{n=0}^{\infty} \widetilde{U}_{n}^{(\alpha)}(x) \frac{t^{n}}{n !}\right)\left(\sum_{n=0}^{\infty} y^{n} \frac{t^{n}}{n !}\right) \\
& =\sum_{n=0}^{\infty} \sum_{l=0}^{n}\left(\begin{array}{c}
n \\
l
\end{array}\right) \widetilde{U}_{n-l}^{(\alpha)}(x) y \frac{t^{n}}{n !} .
\end{aligned}
$$

By comparing the coefficients of both sides, we complete the proof of Theorem 2.2.

We also find the relation of the polynomials, $\widetilde{U}_{n}^{(\alpha)}(x)$, and the numbers, $\widetilde{U}_{n}^{(\alpha)}$.

Theorem 2.3 Let $\alpha, x \in \mathbb{C}$ and $n$ be non-negative integers. Then we get

$$
\widetilde{U}_{n}^{(\alpha)}(x)=\sum_{l=0}^{n}\left(\begin{array}{l}
n \\
l
\end{array}\right) \widetilde{U}_{n-l}^{(\alpha)} x^{l}
$$

Proof This proof is very similar to the proof of Theorem 2.3:

$$
\begin{aligned}
\sum_{n=0}^{\infty} \widetilde{U}_{n}^{(\alpha)}(x) \frac{t^{n}}{n !} & =\left(\frac{e^{t}}{e^{t}+e^{-t}-1}\right)^{\alpha} e^{x t} \\
& =\left(\sum_{n=0}^{\infty} \widetilde{U}_{n}^{(\alpha)} \frac{t^{n}}{n !}\right)\left(\sum_{n=0}^{\infty} x^{n} \frac{t^{n}}{n !}\right)=\sum_{n=0}^{\infty} \sum_{l=0}^{n}\left(\begin{array}{c}
n \\
l
\end{array}\right) \widetilde{U}_{n-l}^{(\alpha)} x^{x} \frac{t^{n}}{n !} .
\end{aligned}
$$


By comparing the coefficients of both sides, we complete the proof of the Theorem 2.3. Of course, we can get a simple proof by substituting $y=0$ in Theorem 2.2.

Theorem 2.4 Let $n, k \in \mathbb{N} \cup\{0\}$ and $k+1 \leq n$. For $\alpha=1$, we have

$$
\widetilde{U}_{n+1}(x)-(1+x) \widetilde{U}_{n}(x)=\sum_{k=0}^{n-1}\left(\begin{array}{l}
n \\
k
\end{array}\right)\left(\left(1-2^{n-k}\right) \widetilde{U}_{k+1}(x)-\left(1+2^{n-k} x-x\right) \widetilde{U}_{k}(x)\right) .
$$

Proof From Definition 2.1, we easily see that the following equation holds true.

Let $\alpha=1$. Then we get

$$
\sum_{n=0}^{\infty} \widetilde{U}_{n}^{(1)}(x) \frac{t^{n}}{n !}=\left(\frac{e^{t}}{e^{t}+e^{-t}-1}\right) e^{t x}=\sum_{n=0}^{\infty} \widetilde{U}_{n}(x) \frac{t^{n}}{n !}
$$

Differentiating with respect to $t$, we find

$$
\sum_{n=1}^{\infty} \widetilde{U}_{n}(x) \frac{t^{n-1}}{(n-1) !}=\frac{2-e^{t}}{\left(e^{t}+e^{-t}-1\right)^{2}} e^{t x}+\frac{e^{t}}{e^{t}+e^{-t}-1} x e^{t x}
$$

If we multiply the above equation throughout by $\left(e^{2 t}-e^{t}+1\right)$, then we have

$$
\begin{aligned}
& \left(e^{2 t}-e^{t}+1\right) \sum_{n=1}^{\infty} \widetilde{U}_{n}(x) \frac{t^{n-1}}{(n-1) !} \\
& =\frac{e^{t}}{e^{t}+e^{-t}-1} e^{t x}\left(2-e^{t}\right)+\frac{e^{t}}{e^{t}+e^{-t}-1} e^{t x}\left(e^{2 t}-e^{t}+1\right) x
\end{aligned}
$$

Then, the left-hand side gets transformed as follows in (2.1):

$$
\begin{aligned}
& \left(e^{2 t}-e^{t}+1\right) \sum_{n=1}^{\infty} \widetilde{U}_{n}(x) \frac{t^{n-1}}{(n-1) !} \\
& \quad=\sum_{n=0}^{\infty}\left(\sum_{k=0}^{n}\left(\begin{array}{l}
n \\
k
\end{array}\right)\left(2^{n-k}-1\right) \widetilde{U}_{k+1}(x)+\widetilde{U}_{n+1}(x)\right) \frac{t^{n}}{n !}
\end{aligned}
$$

And the right-hand side of (2.1) gets transformed in the following form:

$$
\begin{aligned}
& \frac{e^{t}}{e^{t}+e^{-t}-1} e^{t x}\left(2-e^{t}\right)+\frac{e^{t}}{e^{t}+e^{-t}-1} e^{t x}\left(e^{2 t}-e^{t}+1\right) x \\
& =\sum_{n=0}^{\infty}\left((2+x) \widetilde{U}_{n}(x)-\sum_{k=0}^{n}\left(\begin{array}{l}
n \\
k
\end{array}\right)\left(1+2^{n-k} x-x\right) \widetilde{U}_{k}(x)\right) \frac{t^{n}}{n !} .
\end{aligned}
$$

Comparing the coefficients of $\frac{t^{n}}{n !}$ in (2.2) and (2.3), we can represent the equation as

$$
\sum_{k=0}^{n}\left(\begin{array}{l}
n \\
k
\end{array}\right)\left(2^{n-k}-1\right) \widetilde{U}_{k+1}(x)+\widetilde{U}_{n+1}(x)=(2+x) \widetilde{U}_{n}(x)-\sum_{k=0}^{n}\left(\begin{array}{l}
n \\
k
\end{array}\right)\left(1+2^{n-k} x-x\right) \widetilde{U}_{k}(x) .
$$


Rearranging, we get

$$
\widetilde{U}_{n+1}(x)-(1+x) \widetilde{U}_{n}(x)=\sum_{k=0}^{n-1}\left(\begin{array}{l}
n \\
k
\end{array}\right)\left(\left(1-2^{n-k}\right) \widetilde{U}_{k+1}(x)-\left(1+2^{n-k} x-x\right) \widetilde{U}_{k}(x)\right) .
$$

Thus we complete the proof.

Remark From Theorem 2.4, we easily see the following.

If $x=0$ and $\alpha=1$, then

$$
\widetilde{U}_{n+1}-\widetilde{U}_{n}=\sum_{k=0}^{n-1}\left(\begin{array}{l}
n \\
k
\end{array}\right)\left(\left(1-2^{n-k}\right) \widetilde{U}_{k+1}-\widetilde{U}_{k}\right)
$$

If $x=1$ and $\alpha=1$, then

$$
\widetilde{U}_{n+1}(1)-2 \widetilde{U}_{n}(1)=\sum_{k=0}^{n-1}\left(\begin{array}{l}
n \\
k
\end{array}\right)\left(\left(1-2^{n-k}\right) \widetilde{U}_{k+1}(1)-2^{n-k} \widetilde{U}_{k}(1)\right) .
$$

So far, we got some properties of polynomials $\widetilde{U}_{n}^{(\alpha)}(x)$. From now on, we will investigate the relation of the polynomials $\widetilde{U}_{n}^{(\alpha)}(x)$, the Stirling numbers and the central factorial numbers.

Theorem 2.5 Let $n, k, l \in \mathbb{N} \cup\{0\}$ and $n \geq k-l-1$. Then we have

$$
\begin{aligned}
& \widetilde{U}_{2 n}^{(\alpha)}(x)=\sum_{m=0}^{n}\left(\left(\begin{array}{c}
2 n \\
2 m
\end{array}\right) a_{(2(n-m), k)} x^{2 m}+\left(\begin{array}{c}
2 n \\
2 m+1
\end{array}\right) b_{(2(n-m)-1, k)} x^{2 m+1}\right), \\
& \widetilde{U}_{2 n+1}^{(\alpha)}(x)=\sum_{m=0}^{n}\left(\left(\begin{array}{c}
2 n+1 \\
2 m
\end{array}\right) b_{(2(n-m)+1, k)} x^{2 m}+\left(\begin{array}{c}
2 n+1 \\
2 m+1
\end{array}\right) a_{(2(n-m), k)} x^{2 m+1}\right),
\end{aligned}
$$

where

$$
\begin{aligned}
& a_{(2 n, k)}=\sum_{k=0}^{2 n} \sum_{l=0}^{\left[\frac{k}{2}\right]}\left(\begin{array}{c}
2 n \\
2 l
\end{array}\right)(-1)^{k-2 l} \sum_{j=k-2 l}^{n-l} \frac{(2 j) !}{j !} T(n-l, j) s(j, k-2 l) \alpha^{k}, \\
& b_{(2 n+1, k)}=\sum_{k=1}^{2 n+1} \sum_{l=0}^{\left[\frac{k-1}{2}\right]}\left(\begin{array}{c}
2 n+1 \\
2 l-1
\end{array}\right)(-1)^{k-2(l+1)} \sum_{j=k-(2 l+1)}^{n-l} \frac{(2 j) !}{j !} T(n-l, j) s(j, k-(2 l+1)) \alpha^{k},
\end{aligned}
$$

and $[x]$ is the greatest integer not exceeding $x$.

Proof By using the Stirling numbers and the central factorial numbers, we express the polynomials $\widetilde{U}_{n}^{(\alpha)}(x)$ as follows:

$$
\begin{aligned}
\sum_{n=0}^{\infty} \widetilde{U}_{n}^{(\alpha)}(x) \frac{t^{n}}{n !} & =\left(\frac{e^{t}}{e^{t}+e^{-t}-1}\right)^{\alpha} e^{t x}=\left(\frac{1}{e^{t}+e^{-t}-1}\right)^{\alpha} e^{t(\alpha+x)} \\
& =\left(\frac{1}{1+\left(e^{t}+e^{-t}-2\right)}\right)^{\alpha} e^{t(\alpha+x)}
\end{aligned}
$$




$$
\begin{aligned}
& =\sum_{j=0}^{\infty}\left(\begin{array}{c}
j+\alpha-1 \\
j
\end{array}\right)(-1)^{j}\left(e^{t}+e^{-t}-2\right)^{j} e^{t(\alpha+x)} \\
& =\sum_{j=0}^{\infty}(-1)^{j}\left(\begin{array}{c}
\alpha+j-1 \\
j
\end{array}\right)(2 j) ! \sum_{n=j}^{\infty} T(n, j) \frac{t^{2 n}}{(2 n) !} e^{t(\alpha+x)} \\
& =\sum_{n=0}^{\infty} \sum_{j=0}^{n}(-1)^{j}\left(\begin{array}{c}
\alpha+j-1 \\
j
\end{array}\right)(2 j) ! T(n, j) \frac{t^{2 n}}{(2 n) !} e^{t(\alpha+x)} \\
& =\sum_{n=0}^{\infty} \sum_{j=0}^{n} \sum_{k=0}^{j}(-1)^{k} s(j, k) \alpha^{k} \frac{(2 j) !}{j !} T(n, j) \frac{t^{2 n}}{(2 n) !} \sum_{n=0}^{\infty} \alpha^{n} \frac{t^{n}}{n !} \sum_{n=0}^{\infty} x^{n} \frac{t^{n}}{n !} .
\end{aligned}
$$

After some calculation, we get

$$
\begin{aligned}
& \sum_{n=0}^{\infty} \sum_{j=0}^{n} \sum_{k=0}^{j}(-1)^{k} s(j, k) \alpha^{k} \frac{(2 j) !}{j !} T(n, j) \frac{t^{2 n}}{(2 n) !} \sum_{n=0}^{\infty} \alpha^{n} \frac{t^{n}}{n !} \\
& =\sum_{n=0}^{\infty} \sum_{k=0}^{2 n} \sum_{l=0}^{\left[\frac{k}{2}\right]}\left(\begin{array}{c}
2 n \\
2 l
\end{array}\right)(-1)^{k-2 l} \sum_{j=k-2 l}^{n-l} \frac{(2 j) !}{j !} T(n-l, j) s(j, k-2 l) \alpha^{k} \frac{t^{2 n}}{(2 n) !} \\
& \quad+\sum_{n=0}^{\infty} \sum_{k=1}^{2 n+1} \sum_{l=0}^{\left.\frac{[-1}{2}\right]}\left(\begin{array}{c}
2 n+1 \\
2 l-1
\end{array}\right)(-1)^{k-2(l+1)} \\
& \quad \times \sum_{j=k-(2 l+1)}^{n-l} \frac{(2 j) !}{j !} T(n-l, j) s(j, k-(2 l+1)) \alpha^{k} \frac{t^{2 n+1}}{(2 n+1) !},
\end{aligned}
$$

where $[x]$ is the greatest integer not exceeding $x$.

Let

$$
\begin{aligned}
& a_{(2 n, k)}=\sum_{k=0}^{2 n} \sum_{l=0}^{\left[\frac{k}{2}\right]}\left(\begin{array}{c}
2 n \\
2 l
\end{array}\right)(-1)^{k-2 l} \sum_{j=k-2 l}^{n-l} \frac{(2 j) !}{j !} T(n-l, j) s(j, k-2 l) \alpha^{k} \\
& b_{(2 n+1, k)}=\sum_{k=1}^{2 n+1} \sum_{l=0}^{\left[\frac{k-1}{2}\right]}\left(\begin{array}{c}
2 n+1 \\
2 l-1
\end{array}\right)(-1)^{k-2(l+1)} \sum_{j=k-(2 l+1)}^{n-l} \frac{(2 j) !}{j !} T(n-l, j) s(j, k-(2 l+1)) \alpha^{k}
\end{aligned}
$$

Then we have

$$
\sum_{n=0}^{\infty} \widetilde{U}_{n}^{(\alpha)}(x) \frac{t^{n}}{n !}=\left(\sum_{n=0}^{\infty} a_{(2 n, k)} \frac{t^{2 n}}{(2 n) !}+\sum_{n=0}^{\infty} b_{(2 n+1, k)} \frac{t^{2 n+1}}{(2 n+1) !}\right) \sum_{n=0}^{\infty} x^{n} \frac{t^{n}}{n !}
$$

From the above equation, we have to consider odd terms and even terms by using the Cauchy product. Thus, we get generating terms by dividing the odd terms and the even terms, respectively,

$$
\sum_{n=0}^{\infty} \widetilde{U}_{n}^{(\alpha)}(x) \frac{t^{n}}{n !}=\sum_{n=0}^{\infty} \widetilde{U}_{2 n}^{(\alpha)}(x) \frac{t^{2 n}}{(2 n) !}+\sum_{n=0}^{\infty} \widetilde{U}_{2 n+1}^{(\alpha)}(x) \frac{t^{2 n+1}}{(2 n+1) !} .
$$


The following equations represent $\widetilde{U}_{n}^{(\alpha)}(x)$ for $n$ even and $n$ odd terms:

$$
\begin{aligned}
\widetilde{U}_{2 n}^{(\alpha)}(x) & =\sum_{m=0}^{n}\left(\begin{array}{c}
2 n \\
2 m
\end{array}\right) a_{(2(n-m), k)} x^{2 m}+\sum_{m=0}^{n}\left(\begin{array}{c}
2 n \\
2 m+1
\end{array}\right) b_{(2(n-m)-1, k)} x^{2 m+1} \\
& =\sum_{m=0}^{n}\left(\left(\begin{array}{c}
2 n \\
2 m
\end{array}\right) a_{(2(n-m), k)} x^{2 m}+\left(\begin{array}{c}
2 n \\
2 m+1
\end{array}\right) b_{(2(n-m)-1, k)} x^{2 m+1}\right), \\
\widetilde{U}_{2 n+1}^{(\alpha)}(x) & =\sum_{m=0}^{n}\left(\begin{array}{c}
2 n+1 \\
2 m
\end{array}\right) b_{(2(n-m)+1, k)} x^{2 m}+\sum_{m=0}^{n}\left(\begin{array}{c}
2 n+1 \\
2 m+1
\end{array}\right) a_{(2(n-m), k)} x^{2 m+1} \\
& =\sum_{m=0}^{n}\left(\left(\begin{array}{c}
2 n+1 \\
2 m
\end{array}\right) b_{(2(n-m)+1, k)} x^{2 m}+\left(\begin{array}{c}
2 n+1 \\
2 m+1
\end{array}\right) a_{(2(n-m), k)} x^{2 m+1}\right),
\end{aligned}
$$

where

$$
\begin{aligned}
& a_{(2 n, k)}=\sum_{k=0}^{2 n} \sum_{l=0}^{\left[\frac{k}{2}\right]}\left(\begin{array}{c}
2 n \\
2 l
\end{array}\right)(-1)^{k-2 l} \sum_{j=k-2 l}^{n-l} \frac{(2 j) !}{j !} T(n-l, j) s(j, k-2 l) \alpha^{k}, \\
& b_{(2 n+1, k)}=\sum_{k=1}^{2 n+1} \sum_{l=0}^{\left.\frac{k-1}{2}\right]}\left(\begin{array}{c}
2 n+1 \\
2 l-1
\end{array}\right)(-1)^{k-2(l+1)} \sum_{j=k-(2 l+1)}^{n-l} \frac{(2 j) !}{j !} T(n-l, j) s(j, k-(2 l+1)) \alpha^{k},
\end{aligned}
$$

and $[x]$ is the greatest integer not exceeding $x$.

Thus, we complete the proof of Theorem 2.5.

From Theorem 2.5, we find examples of the $\widetilde{U}_{n}^{(\alpha)}(x)$ polynomials.

Example 2.6 Let $n=1,2,3,4,5,6,7,8$. Then we have

$$
\begin{aligned}
& a_{(2, k)}=\alpha^{2}-2 \alpha, \\
& a_{(4, k)}=\alpha^{4}-12 \alpha^{3}+12 \alpha^{2}+10 \alpha, \\
& a_{(6, k)}=\alpha^{6}-30 \alpha^{5}+180 \alpha^{4}+30 \alpha^{3}-300 \alpha^{2}-182 \alpha, \\
& \ldots
\end{aligned}
$$

Also,

$$
\begin{aligned}
& b_{(1, k)}=\alpha, \\
& b_{(3, k)}=\alpha^{3}-6 \alpha^{2}, \\
& b_{(5, k)}=\alpha^{5}-20 \alpha^{4}+60 \alpha^{3}+50 \alpha^{2}, \\
& \cdots .
\end{aligned}
$$

Therefore, we can express $\widetilde{U}_{n}^{(\alpha)}(x)$ as a function of $x$ and $\alpha$ explicitly. For instance,

$$
\begin{aligned}
& \widetilde{U}_{1}^{(\alpha)}(x)=x+\alpha, \\
& \widetilde{U}_{2}^{(\alpha)}(x)=x^{2}-2 \alpha x+\alpha^{2}-2 \alpha,
\end{aligned}
$$




$$
\widetilde{U}_{3}^{(\alpha)}(x)=x^{3}+3 \alpha x^{2}+3 \alpha^{2} x-6 \alpha x+\alpha^{3}-6 \alpha^{2},
$$

\section{Some relations of the polynomials $\tilde{U}_{n}^{(\alpha)}(x)$, the Euler polynomials, and the Genocchi polynomials}

In this chapter, we find interesting relations between the polynomials $\widetilde{U}_{n}^{(\alpha)}(x)$, the Euler polynomials, and the Genocchi polynomials. In other words, the polynomials $\widetilde{U}_{n}^{(\alpha)}(x)$ can be shown in a combined form by using Euler numbers and polynomials. We also easily see the polynomials $\widetilde{U}_{n}^{(\alpha)}(x)$ that are represented by Genocchi numbers and polynomials.

First, we study a link between the polynomials $\widetilde{U}_{n}^{(\alpha)}(x)$ and the Euler polynomials $E_{n}^{(\alpha)}(x)$.

Theorem 3.1 Let $\alpha \in \mathbb{C}$, $n$ be a non-negative integer. Then we have

$$
2^{\alpha} \widetilde{U}_{n}^{(\alpha)}(x)=(-3)^{n}\left(E_{n}^{(\alpha)}\left(-\frac{1}{3} x\right)+E_{n}^{(\alpha)}\left(-\frac{1}{3} x+\frac{1}{3} \alpha\right)\right) .
$$

Proof From Definition 2.1, we have

$$
\begin{aligned}
\sum_{n=0}^{\infty} \widetilde{U}_{n}^{(\alpha)}(x) \frac{t^{n}}{n !} & =\left(\frac{e^{t}}{e^{t}+e^{-t}-1}\right)^{\alpha} e^{t x} \\
& =\frac{1}{2^{\alpha}}\left(\left(\frac{2}{1+e^{-3 t}}\right)^{\alpha} e^{t x}+\left(\frac{2}{1+e^{-3 t}}\right)^{\alpha} e^{t(x-\alpha)}\right) \\
& =\sum_{n=0}^{\infty}\left(\frac{(-3)^{n}}{2^{\alpha}}\left(E_{n}^{(\alpha)}\left(-\frac{1}{3} x\right)+E_{n}^{(\alpha)}\left(-\frac{1}{3} x+\frac{1}{3} \alpha\right)\right)\right) \frac{t^{n}}{n !} .
\end{aligned}
$$

By comparing the coefficients of both sides, we have

$$
\widetilde{U}_{n}^{(\alpha)}(x)=\frac{(-3)^{n}}{2^{\alpha}}\left(E_{n}^{(\alpha)}\left(-\frac{1}{3} x\right)+E_{n}^{(\alpha)}\left(-\frac{1}{3} x+\frac{1}{3} \alpha\right)\right) .
$$

Hence the proof of the Theorem 3.1 is complete.

From Theorem 3.1, we easily get the following corollary.

Corollary 3.2 Let $\alpha=1$. Then one has

$$
\begin{aligned}
2 \widetilde{U}_{n}(x) & =(-3)^{n}\left(E_{n}\left(-\frac{1}{3} x\right)+E_{n}\left(-\frac{1}{3} x+\frac{1}{3}\right)\right) \\
& =(-3)^{n}\left(E_{n}\left(-\frac{1}{3} x\right)+(-1)^{n} E_{n}\left(-\frac{1}{3} x+\frac{2}{3}\right)\right) .
\end{aligned}
$$

Proof Let $\alpha=1$ in Definition 2.1 and Theorem 3.1, respectively. Then we easily obtain the proof of Corollary 3.2.

Theorem 3.3 Let $n, v_{1}, \ldots, v_{k}, k \in \mathbb{N}$. Then we have

$$
\widetilde{U}_{n}^{(k)}(x)=\frac{3^{n-l}}{2^{k}} \sum_{l=0}^{n}(n-l) ! \sum_{\substack{v_{1}, \ldots, v_{k}=0 \\ v_{1}+\cdots+v_{k}=n-l}} \frac{\left(E_{v_{1}}\left(\frac{2}{3} x\right)+(-1)^{v_{1}} E_{v_{1}}\right) \cdots\left(E_{v_{k}}\left(\frac{2}{3} x\right)+(-1)^{v_{k}} E_{v_{k}}\right)}{v_{1} ! \cdots v_{k} !} x^{l} .
$$


Proof From the Definition 2.1 and the Euler numbers and polynomials, we can write the following equation:

$$
\begin{aligned}
\sum_{n=0}^{\infty} \widetilde{U}_{n}^{(k)}(x) \frac{t^{n}}{n !} & =\left(\frac{e^{t}}{e^{t}+e^{-t}-1}\right)^{k} e^{t x} \\
& =\left(\frac{1}{1+e^{-3 t}}+\frac{1}{1+e^{3 t}} e^{2 t}\right)^{k} e^{t x} \\
& =\frac{1}{2^{k}}\left(\sum_{n=0}^{\infty}\left(3^{n} E_{n}\left(\frac{2}{3} x\right)+(-3)^{n} E_{n}\right) \frac{t^{n}}{n !}\right)^{k} \sum_{n=0}^{\infty} x^{n} \frac{t^{n}}{n !} .
\end{aligned}
$$

Here, we can represent the above right-hand side as the following equation:

$$
\begin{aligned}
& \frac{1}{2^{k}}\left(\sum_{n=0}^{\infty}\left(3^{n} E_{n}\left(\frac{2}{3} x\right)+(-3)^{n} E_{n}\right) \frac{t^{n}}{n !}\right)^{k} \\
& \quad=\frac{1}{2^{k}} \sum_{n=0}^{\infty} \sum_{\substack{v_{1}, \ldots, v_{k}=0 \\
v_{1}+\cdots+v_{k}=n}} \frac{3^{n}\left(E_{v_{1}}\left(\frac{2}{3} x\right)+(-1)^{v_{1}} E_{v_{1}}\right) \cdots\left(E_{v_{k}}\left(\frac{2}{3} x\right)+(-1)^{v_{k}} E_{v_{k}}\right)}{v_{1} ! \cdots v_{k} !} .
\end{aligned}
$$

Therefore, we obtain

$$
\widetilde{U}_{n}^{(k)}(x)=\frac{3^{n-l}}{2^{k}} \sum_{l=0}^{n}(n-l) ! \sum_{\substack{v_{1}, \ldots, v_{k}=0 \\ v_{1}+\cdots+v_{k}=n-l}} \frac{\left(E_{v_{1}}\left(\frac{2}{3} x\right)+(-1)^{v_{1}} E_{v_{1}}\right) \cdots\left(E_{v_{k}}\left(\frac{2}{3} x\right)+(-1)^{v_{k}} E_{v_{k}}\right)}{v_{1} ! \cdots v_{k} !} x^{l} .
$$

Secondly, we note a link between the polynomials $\widetilde{U}_{n}^{(\alpha)}(x)$, the Genocchi numbers, $G_{n}^{(\alpha)}$, and the polynomials $G_{n}^{(\alpha)}(x)$.

Theorem 3.4 Let $k, n \in \mathbb{N}$. Then we have

$$
2^{k} k !\left(\begin{array}{c}
k+n \\
k
\end{array}\right) \widetilde{U}_{n}^{(k)}(x)=(-3)^{n}\left(G_{k+n}^{(k)}\left(-\frac{1}{3} x\right)+G_{k+n}^{(k)}\left(-\frac{1}{3} x+\frac{1}{3} k\right)\right) .
$$

Proof $\widetilde{U}_{n}^{(k)}(x)$ is represented as follows:

$$
\begin{aligned}
\sum_{n=0}^{\infty} \widetilde{U}_{n}^{(k)}(x) \frac{t^{n}}{n !} & =\frac{1}{(2 t)^{k}}\left(\frac{2 t}{1+e^{-3 t}}\right)^{k} e^{t x}+\frac{1}{(2 t)^{k}}\left(\frac{2 t}{1+e^{-3 t}}\right)^{k} e^{t(x-k)} \\
& =\frac{1}{(-6 t)^{k}} \sum_{n=0}^{\infty}(-3)^{n}\left(G_{n}^{(k)}\left(-\frac{1}{3} x\right)+G_{n}^{(k)}\left(-\frac{1}{3} x+\frac{1}{3} k\right)\right) \frac{t^{n}}{n !} \\
& =\frac{1}{(-6)^{k}} \sum_{n=0}^{\infty}(-3)^{n}\left(G_{n}^{(k)}\left(-\frac{1}{3} x\right)+G_{n}^{(k)}\left(-\frac{1}{3} x+\frac{1}{3} k\right)\right) \frac{t^{n-k}}{n !} .
\end{aligned}
$$

By comparing the coefficients of both sides in the above equation, we derive

$$
\left.\widetilde{U}_{n}^{(k)}(x)=\frac{(-3)^{n}}{2^{k} k !\left(\begin{array}{c}
\left(\begin{array}{c}
n \\
k
\end{array}\right) \\
(k+n
\end{array}\right.}\left(-\frac{1}{3} x\right)+G_{k+n}^{(k)}\left(-\frac{1}{3} x+\frac{1}{3} k\right)\right) .
$$

Thus we complete the proof of Theorem 3.4. 
From Theorem 3.4, we get the following corollary.

Corollary 3.5 Let $k=1, n \in \mathbb{N}$ and $k+1 \leq n$. Then we derive

$$
\begin{aligned}
2(n+1) \widetilde{U}_{n}(x) & =(-3)^{n}\left(G_{n+1}\left(-\frac{1}{3} x\right)+G_{n+1}\left(-\frac{1}{3} x+\frac{1}{3}\right)\right) \\
& =(-3)^{n}\left(G_{n+1}\left(-\frac{1}{3} x\right)+(-1)^{n} G_{n+1}\left(-\frac{1}{3} x+\frac{2}{3}\right)\right) .
\end{aligned}
$$

Proof Let $\alpha=1, k=1$ in Definition 2.1 and Theorem 3.4, respectively. Then we easily obtain the proof of Corollary 3.5.

\section{Competing interests}

The authors declare that they have no competing interests.

\section{Authors' contributions}

All authors contributed equally to the manuscript and read and approved the final manuscript.

\section{Acknowledgements}

The authors express their gratitude to the referee for his/her valuable comments. This work was supported by NRF (National Research Foundation of Korea) Grant funded by the Korean Government (NRF-2013-Fostering Core Leaders of the Future Basic Science Program)

\section{Received: 28 September 2013 Accepted: 26 December 2013 Published: 24 Jan 2014}

\section{References}

1. Kim, T: Carlitz q-Bernoulli numbers and q-Stirling numbers. Number Theory. arXiv:0708.3306

2. Srivastava, HM, Liu, GD: Some identities and congruences involving a certain family of numbers. Russ. J. Math. Phys. $16,536-542(2009)$

3. Liu, GD, Zhang, WP: Applications of an explicit formula for the generalized Euler numbers. Acta Math. Sin. Engl. Ser. 24(2), 343-352 (2008)

4. Sun, ZH: Identities and congruences for a new sequence. Int. J. Number Theory 1(8), 207-225 (2012)

5. Sun, ZH: Some properties of a sequence analogous to Euler numbers. Bull. Aust. Math. Soc. 87, 425-440 (2013)

6. Kim, DS, Kim, T, Kim, YH, Lee, SH: Some arithmetic properties of Bernoulli and Euler numbers. Adv. Stud. Contemp. Math. 4(22), 467-480 (2012)

7. Kim, M-S, Lee, JH: On sums of products of the extended q-Euler numbers. J. Math. Anal. Appl. 397, $522-528$ (2013)

8. Kim, M-S: On Euler numbers, polynomials and related $p$-adic integrals. J. Number Theory 129, 2166-2179 (2009)

9. Kim, T: New approach to q-Euler polynomials of higher order. Russ. J. Math. Phys. 2(17), $218-225$ (2010)

10. Ryoo, CS: A note on the weighted q-Euler numbers and polynomials. Adv. Stud. Contemp. Math. 21, 47-54 (2011)

11. Ryoo, CS: A numerical computation on the structure of the roots of $q$-extension of Genocchi polynomials. Appl. Math. Lett. 4(21), 348-354 (2008)

12. Ryoo, CS, Kim, T, Jang, L-C: Some relationships between the analogs of Euler numbers and polynomials. J. Inequal. Appl. 2007, Article ID 86052 (2007). doi:10.1155/2007/86052

13. Cangul, IN, Ozden, H, Simsek, Y: A new approach to $q$-Genocchi numbers and their interpolation functions. Nonlinear Anal., Theory Methods Appl. 71, 793-799 (2009)

14. Rim, SH, Park, KH, Moon, EJ: A note on the 9 -Genocchi numbers and polynomials. J. Inequal. Appl. 2007, Article ID $71452(2007)$

15. Simsek, Y, Cangul, IN, Kurt, V, Kim, D: q-Genocchi numbers and polynomials associated with q-Genocchi-type L-functions. Adv. Differ. Equ. 2008, Article ID 815750 (2008)

16. Kurt, V, Cenkci, M: A new approach to $q$-Genocchi numbers and polynomials. Bull. Korean Math. Soc. 3(47), 575-583 (2010). doi:10.4134/BKMS.2010.47.3.575 